\title{
Experiência Inicial em Málaga (Espanha) com Prótese Aórtica CoreValve para Tratamento de Estenose Aórtica Sintomática Grave
}

\author{
José M. Hernández-García ${ }^{1}$, Antonio J. Muñoz García', Juan H. Alonso-Briales', \\ Manuel F. Jiménez-Navarro', Antonio J. Domínguez-Franco ${ }^{1}$, Isabel Rodríguez-Bailón' ${ }^{1}$, \\ Paula Hernández¹, Eduardo Olalla-Mercadé1, Eduardo de Teresa-Galván
}

\section{RESUMO}

Introdução: A prevalência de estenose aórtica grave vem crescendo em decorrência do aumento da expectativa de vida. Alguns pacientes não se beneficiam do tratamento cirúrgico por causa das comorbidades associadas. Apresentamos os resultados de nossa experiência no tratamento percutâneo da estenose aórtica. Método: Estudo prospectivo, realizado entre abril de 2008 e fevereiro de 2009, em que 31 pacientes consecutivos com estenose aórtica grave sintomática e que apresentavam alto risco cirúrgico foram tratados com a prótese aórtica CoreValve. Todos os procedimentos foram realizados com anestesia local. Em 29 casos a via de acesso foi a artéria femoral, com introdutor $18 \mathrm{~F}$, a punção femoral foi fechada com Prostar $10 \mathrm{~F}$, e em 2 pacientes a artéria subclávia esquerda foi a via de acesso. Resultados: A média de idade foi de $77,8 \pm 8,9$ anos e o EuroSCORE logístico médio foi de 17,5 $\pm 12,6 \%$. O sucesso do implante foi de $100 \%$. O gradiente de pico a pico após o implante desapareceu. Nenhum paciente apresentou insuficiência aórtica residual $>$ grau 2 de Sellers. A mortalidade aos 30 dias foi de $3,2 \%$. Foram necessários marca-passos definitivos em $31 \%$ dos pacientes. Depois de acompanhamento médio de $154 \pm 90$ dias, foram registrados 4 óbitos (1 morte súbita e 3 por causas não-cardíacas). Conclusões: $O$ implante percutâneo da prótese aórtica CoreValve como tratamento alternativo da estenose aórtica grave em pacientes com alto risco cirúrgico é factível e seguro, com elevada taxa de êxito no procedimento e porcentual de complicações abaixo do esperado em função do risco cirúrgico estimado com o EuroSCORE.

DESCRITORES: Estenose da valva aórtica. Valvas cardíacas. Implante de prótese de valva/métodos. Próteses valvulares cardíacas.

\author{
ABSTRACT

Malaga (Spain) Early Experience with the
CoreValve Aortic Prosthesis for the Treatment
of Severe Symptomatic Aortic Stenosis

Background: The prevalence of severe aortic stenosis is increasing with the rise in life expectancy. Some patients cannot undergo surgical treatment of aortic stenosis due to associated disorders. We present the results of our experience with the percutaneous treatment of aortic stenosis as an alternative to surgery in high-risk patients. Methods: This prospective cohort study included 31 consecutive high surgical risk patients with severe and symptomatic aortic stenosis treated with a CoreValve aortic prosthesis from April 2008 to February 2009. All the procedures were undertaken with local anesthesia. In 29 patients we used a femoral artery access, with an $18 \mathrm{~F}$ introducer, closing the femoral puncture with a $10 \mathrm{~F}$ Prostar, and in two patients the access was via the left subclavian artery. Results: The mean age of the patients was $77.8 \pm 8.9$ years and the mean logistic EuroSCORE was $17.5 \pm 12.6 \%$. The implant was successful in $100 \%$ of cases. The peak-to-peak gradient after the implant disappeared. No patient had residual aortic insufficiency Sellers' grade $>2$. One-month mortality was $3.2 \%$. A definitive pacemaker was required in $31 \%$ of the patients. After a mean follow-up of $154 \pm 90$ days there were four deaths ( 1 sudden death and 3 for noncardiac causes). Conclusions: Percutaneous implantation of the CoreValve aortic prosthesis as an alternative treatment for severe aortic stenosis in high surgical risk patients is feasible and safe, with a high success rate for the procedure and a percentage of complications below the expected for the surgical risk, as estimated by the EuroSCORE.

DESCRIPTORS: Aortic valve stenosis. Heart valves. Heart valve prosthesis implantation/methods. Heart valve prosthesis.

\footnotetext{
1 Hospital Clínico Universitário Virgen de la Victoria - Málaga, Espanha.

Correspondência: Antonio J. Muñoz-García. Servicio de Cardiologia - Hospital Clinico Universitario Virgen de la Victoria - Campus de Teatinos, s/n - Málaga, España - E-29010

E-mail: ajmunozgarcia@secardiologia.es

Recebido em: 10/4/2009 • Aceito em: 11/6/2009
} 
A doença valvar é um problema de saúde importante, estando sua prevalência e seu prognóstico intimamente ligados ao aumento da expectativa de vida ${ }^{1}$. A doença valvar mais frequente na Europa é a estenose aórtica degenerativa grave, predominante em idades avançadas e em pacientes com doenças concomitantes ${ }^{2}$.

O tratamento preferido para pacientes com estenose aórtica grave sintomática é a substituição da valva, que resulta em melhor sobrevida e maior qualidade de vida. Entretanto, foram identificados alguns fatores que levam a maior risco de mortalidade operatória, como em pacientes com fração de ejeção ventricular esquerda reduzida, em que a taxa de mortalidade aumenta de $10 \%$ a $30 \%$. Além disso, a idade avançada ou a presença de doenças associadas aumenta bastante o risco periprocedimento ${ }^{3-6}$ e limita essa opção terapêutica. Pelo menos 33\% dos pacientes com estenose aórtica grave não são indicados para terapia de substituição da valva por terem outros distúrbios subjacentes, conforme dados do Euro Heart Survey ${ }^{7}$. A valvuloplastia aórtica com balões surgiu como tratamento para esses pacientes, embora atualmente se encontre em desuso, pela elevada taxa de reestenose precoce $^{2}$.

A necessidade de novas opções terapêuticas alternativas para pacientes com estenose aórtica que apresentam elevado risco cirúrgico levou à elaboração de um tratamento menos invasivo: a substituição percutânea da valva aórtica. Desde que Cribier et al. ${ }^{8}$ realizaram o primeiro implante humano, mais de 5 mil pacientes já foram tratados com uma das duas próteses biológicas percutâneas disponíveis, a prótese EdwardsSapiens (Edwards Lifesciences, Irvine, Estados Unidos) e a prótese aórtica CoreValve Revalving ${ }^{\mathrm{TM}}$ (CoreValve, Irvine, Estados Unidos) ${ }^{9}$.

Apresentamos os resultados iniciais de nossa experiência no tratamento percutâneo da estenose aórtica Como uma alternativa à cirurgia em pacientes de alto risco.

\section{MÉTODO}

\section{População do estudo}

Entre abril de 2008 e fevereiro de 2009, foi realizado um estudo de coorte prospectivo em nosso centro, que incluiu 31 pacientes consecutivos com estenose aórtica grave sintomática apresentando elevado risco cirúrgico ou que não quiseram se submeter a cirurgia. Todos os pacientes foram avaliados por uma equipe multidisciplinar (cardiologistas clínicos, cardiologistas intervencionistas e cirurgiões cardíacos). Os seguintes estudos foram realizados antes do procedimento para avaliar a elegibilidade dos pacientes: coronariografia, aortografia com injeções no território íleo-femoral, e ecocardiograma transtorácico. Quando esse procedimento não se mostrava conclusivo, era feito também um ecocardiograma transesofágico. O risco cirúrgico foi estimado pelo EuroSCORE logístico.
Os pacientes foram considerados de alto risco cirúrgico quando a equipe multidisciplinar concordava que a cirurgia convencional estaria associada a morbidade e mortalidade excessivas.

Os critérios de inclusão foram os seguintes: 1) estenose grave da valva aórtica com área $<1 \mathrm{~cm}^{2}$ ou $<0,6 \mathrm{~cm}^{2} / \mathrm{m}^{2}$ com ou sem regurgitação da valva aórtica; 2) diâmetro do anel da valva aórtica $\geq 20 \mathrm{~mm}$ e $\leq 27 \mathrm{~mm}$ ao ecocardiograma; e 3) diâmetro da aorta ascendente $\leq 40 \mathrm{~mm}$ (dispositivo de $26 \mathrm{~mm}$ ) ou $\leq 43 \mathrm{~mm}$ (dispositivo de $29 \mathrm{~mm}$ ). Os critérios de exclusão incluíram: hipersensibilidade ou contraindicação a qualquer medicamento do estudo, sepse ou endocardite ativa, infarto do miocárdio ou derrame recente (últimos 30 dias), intervenção coronária percutânea recente (a menos de um mês do procedimento), regurgitação grave da valva mitral ou tricúspide, aneurisma aórtico, diátese hemorrágica ou distúrbio de coagulação, clearance de creatinina $<20 \mathrm{ml} / \mathrm{min}$ ou fração de ejeção ventricular esquerda $<20 \%$.

\section{Descrição do dispositivo}

Neste estudo foi utilizada a terceira geração do CoreValve. O sistema do dispositivo compreende os seguintes componentes:

1. Prótese aórtica CoreValve, que consiste numa válvula de tecido de pericárdio porcino bioprotética trifolheada, montada e suturada em stent de nitinol autoexpansível. A parte inferior da prótese tem elevada força radial para expandir e excluir os folhetos calcificados e evitar retração. A parte média é comprimida para evitar as artérias coronárias, enquanto a parte superior é destacada para centralizar e fixar bem a estrutura do stent na aorta ascendente e propiciar estabilidade longitudinal e posicionamento coaxial. O dispositivo é encontrado em dois tamanhos diferentes, de acordo com a dimensão anelar: a prótese de $26 \mathrm{~mm}$ para valvas aórticas com tamanhos anelares entre $20 \mathrm{~mm}$ e $23 \mathrm{~mm}$, e a prótese de $29 \mathrm{~mm}$ para valvas aórticas com tamanhos anelares entre $24 \mathrm{~mm}$ e $27 \mathrm{~mm}$ (Figura 1).

2. Sistema de liberação com cateter $18 \mathrm{~F}$.

3. Sistema de carregamento descartável, para ser usado uma única vez.

\section{Procedimento}

O procedimento, em todos os casos, foi realizado no laboratório de hemodinâmica, com anestesia local combinada a sedação superficial. A profilaxia antibiótica foi feita com cefalosporina ou com vancomicina, no caso de pacientes alérgicos a betalactâmicos. Em 29 pacientes, a via de acesso foi a artéria femoral, com introdutor de $18 \mathrm{~F}$, e em 2 pacientes a artéria subclávia esquerda foi a via de acesso em colaboração com o cirurgião (em decorrência de doença arterial periférica 
aorto-íleo-femoral ou de diâmetro da artéria ilíaca/femoral $<6 \mathrm{~mm}$ ).

Depois de implantar um cateter marca-passo através da veia jugular, a artéria femoral foi puncionada para que a prótese fosse avançada e colocado um dispositivo de fechamento vascular (Prostar XL $\mathrm{XL}^{\mathrm{TM}}$, Abbott Laboratories, Illinois, Estados Unidos) (Figura 2). Essa punção foi feita sob fluoroscopia, injetando contraste por um cateter introduzido na artéria femoral contralateral. Naqueles pacientes que não tinham sido submetidos a valvuloplastia aórtica no mês anterior, foi realizada valvuloplastia com estimulação cardíaca com marca-passo a $180 \mathrm{bpm}$, a fim de evitar o deslocamento do balão. Os balões usados foram os Numed ${ }^{\mathrm{TM}}$ (NuMed, Rockville, Estados Unidos) de $20 \mathrm{~mm}$ ou $22 \mathrm{~mm}$, para implantes de válvulas pequenas, ou de $25 \mathrm{~mm}$, para

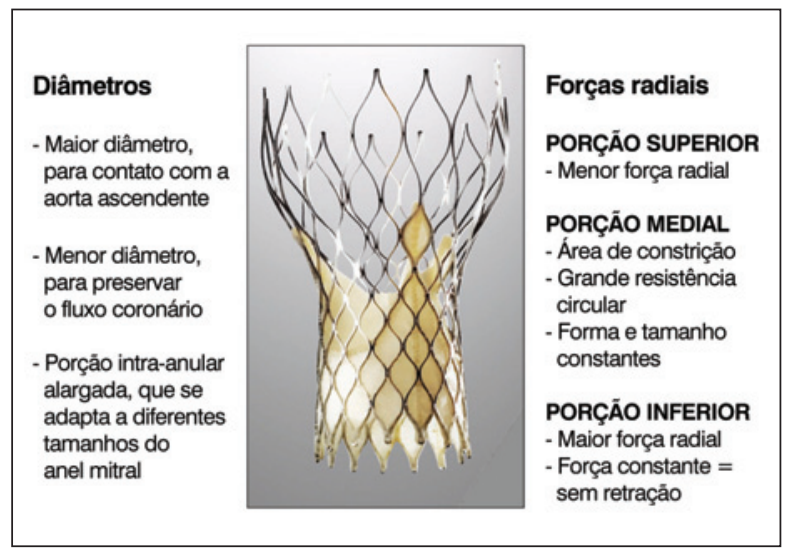

Figura 1 - Terceira geração da prótese aórtica CoreValve Revalving ${ }^{\mathrm{TM}}$. A estrutura autoexpansível tem formato truncado e apresenta três níveis distintos, com diferentes características e funções.

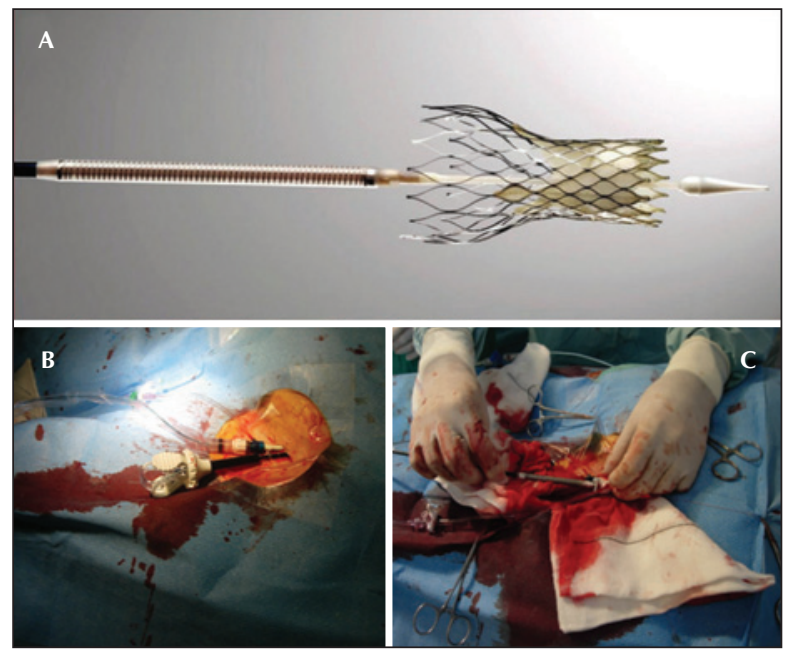

Figura 2 - Em A, prótese da válvula aórtica carregada no cateter. Em B, punção da artéria femoral e posicionamento do dispositivo para fechamento vascular (Prostar XLTM). Em C, prótese sendo avançada através do Prostar. implantes de válvulas maiores. O dispositivo foi então liberado via retrógada sob orientação fluoroscópica e aortográfica (Figura 3). Após o implante, foi medido o gradiente entre ventrículo esquerdo e aorta, sendo observada a presença de insuficiência aórtica residual. O procedimento foi encerrado com o fechamento percutâneo de ambas as artérias femorais. O Prostar XL ${ }^{\mathrm{TM}}$ implantado previamente foi usado para fechar a artéria femoral, e o Angio-Seal ${ }^{\mathrm{TM}}$ (St. Jude Medical, Inc., Minnetonka, Estados Unidos) foi utilizado para fechar a artéria femoral contralateral. Quando se utilizou o acesso pela via subclávia, a artéria foi cirurgicamente exposta e depois puncionada pela técnica de Seldinger, sendo o procedimento realizado da mesma maneira que no acesso femoral. Ao final do procedimento, a artéria era fechada cirurgicamente.

Foi feito controle por ecocardiograma após a intervenção e 72 horas mais tarde, para aferir o desempenho da válvula.

\section{Antiplaquetários e antitrombóticos}

$\mathrm{Na}$ ausência de qualquer contraindicação, administrou-se ácido acetilsalicílico (100 mg/dia) antes do procedimento e indefinidamente a partir de então. Além disso, todos os pacientes receberam clopidogrel, com dose de ataque de $300 \mathrm{mg}$ seguida por $75 \mathrm{mg} / \mathrm{dia}$, por, no mínimo, 6 a 12 meses. Durante a intervenção, o paciente recebeu heparina intravenosa ajustada por peso para obter tempo de coagulação ativada $>250$ segundos.

\section{Acompanhamento}

Todos os pacientes tiveram acompanhamento clínico e eletrocardiográfico aos 30 dias e periodicamente a partir de então (3, 6 e 12 meses). O período médio de acompanhamento foi de $154 \pm 90$ dias.

\section{Definições}

- Sucesso do procedimento: implante adequado e função normal da prótese sem morte periprocedural.

- Eventos cardiovasculares: presença de morte, acidente vascular cerebral ou infarto do miocárdio durante o primeiro mês após o procedimento.

- Complicações vasculares: dissecção da aorta, falha do dispositivo para fechamento percutâneo, ruptura da artéria ilíaca ou femoral, hemorragia requerendo cirurgia.

- Mortalidade $\leq 1$ mês: morte por qualquer causa durante a internação hospitalar ou no primeiro mês após o procedimento.

- Mortalidade > 1 mês: morte por qualquer causa a partir de um mês após o procedimento.

- Mortalidade geral: soma das duas mortalidades. 

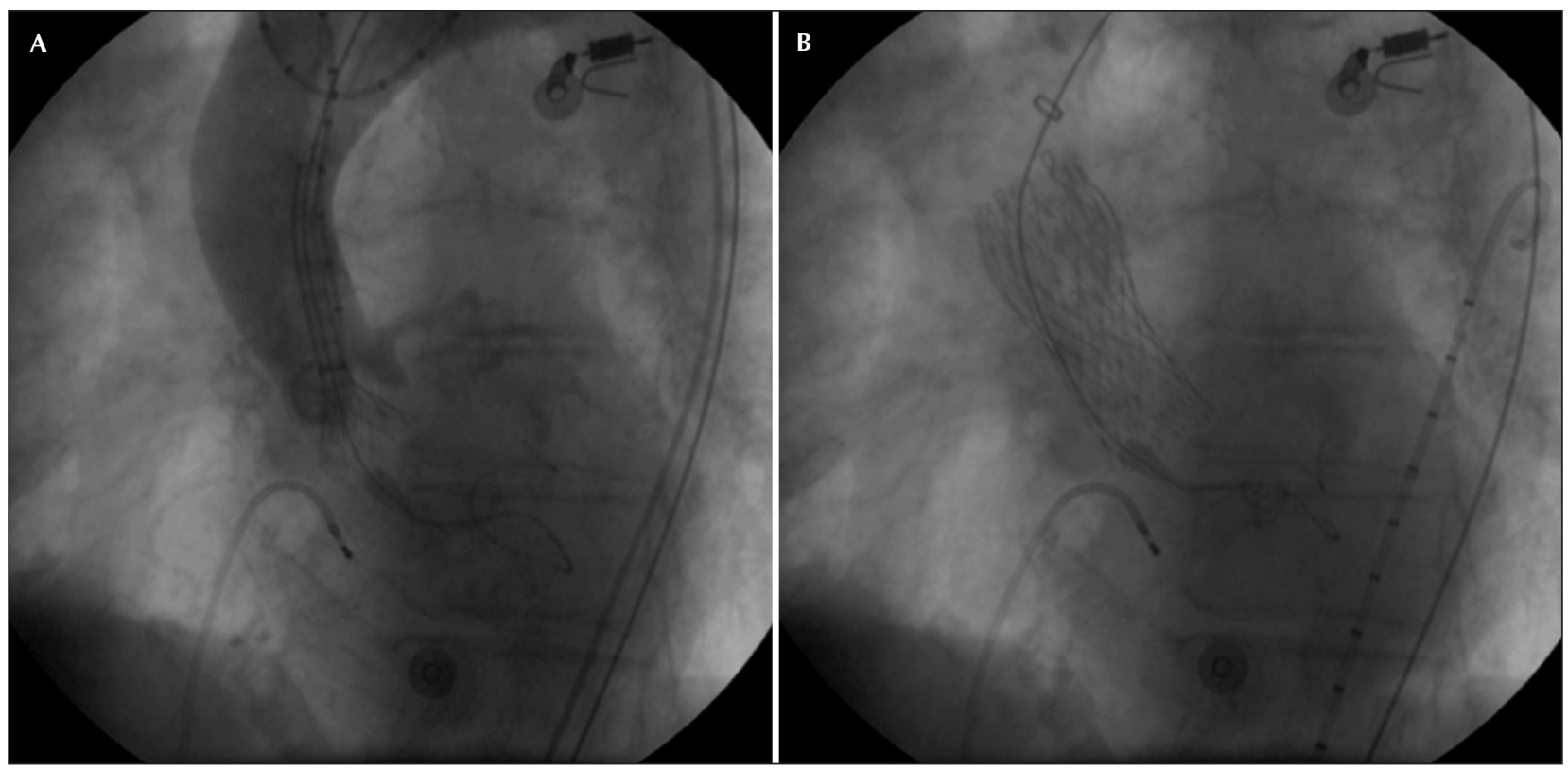

Figura 3 - Sequência angiográfica do implante da prótese aórtica CoreValve. Em A, fase inicial da liberação da prótese sob visualização angiográfica. Em B, resultado da prótese implantada.

\section{Análise estatística}

As variáveis categóricas são apresentadas como frequências e foram comparadas pelo teste de quiquadrado. As variáveis contínuas são apresentadas como média \pm desvio padrão. Foi realizada uma análise estatística descritiva e um estudo de sobrevida pelo método de Kaplan-Meier. Um valor de $\mathrm{P}<0,05$ foi considerado estatisticamente significante. As análises foram realizadas com a versão SPSS 12.0 (SPSS, Inc., Chicago, Estados Unidos).

\section{RESULTADOS}

\section{População de pacientes}

Entre abril de 2008 e fevereiro de 2009, 31 pacientes sintomáticos (19 mulheres e 12 homens), com média de idade de 77,8 \pm 8,9 anos, foram tratados com prótese aórtica CoreValve. As características basais detalhadas dos pacientes são apresentadas na Tabela 1. Um paciente apresentou estenose aórtica em decorrência de implante de prótese biológica disfuncional três anos antes. A área valvar aórtica média foi de $0,33 \mathrm{~cm}^{2}$, com gradiente sistólico máximo de $95 \mathrm{mmHg}$ e gradiente médio de $73 \mathrm{mmHg}$. Outro paciente apresentou dupla lesão aórtica, estenose moderada e regurgitação grave. O restante dos pacientes apresentou estenose aórtica sintomática grave, com gradiente de pressão transvalvar médio de $50 \pm 14 \mathrm{mmHg}(20-93 \mathrm{mmHg})$, e a área valvar aórtica média avaliada por ecocardiograma foi de $0,63 \pm 0,20 \mathrm{~cm}^{2}$. O EuroSCORE médio calculado da população do estudo foi de 17,5 \pm 12,6\% (7$62,6 \%$ ) e $93,6 \%$ dos pacientes estavam nas classes funcionais III ou IV da New York Heart Association.
TABELA 1

Características basais da população do estudo

\begin{tabular}{lc}
\hline Idade, anos & $77,8 \pm 8,9$ \\
Sexo (feminino) & $19(61,3 \%)$ \\
IMC, kg/m² & $29,5 \pm 5,8$ \\
Classe funcional da NYHA & \\
Classe II & $2(6,5 \%)$ \\
Classe III & $18(58,1 \%)$ \\
Classe IV & $11(35,5 \%)$ \\
Cirurgia valvar prévia & $2(6,5 \%)$ \\
Doença coronária & $13(41,9 \%)$ \\
Revascularização cirúrgica prévia & $6(19,4 \%)$ \\
ATPC prévia & $7(22,6 \%)$ \\
Logistic EuroSCORE, \% & $17,5 \pm 12,6$ \\
Fatores de risco cardiovascular & \\
Diabetes melito & $13(41,9 \%)$ \\
Hipercolesterolemia & $19(61,3 \%)$ \\
Hipertensão & $27(87,1 \%)$ \\
Fumo & $9(29 \%)$ \\
Parâmetros ecocardiográficos & \\
Gradiente máximo, mmHg & $76,6 \pm 18$ \\
Gradiente médio, mmHg & $50 \pm 14$ \\
Área da valva aórtica, cm² & $0,63 \pm 0,20$ \\
Anel aórtico, mm & $22,2 \pm 1,6$ \\
Função ventricular esquerda, \% & $62,3 \pm 14,3$ \\
\hline
\end{tabular}

Os dados são expressos em média \pm desvio padrão para variáveis contínuas e em números (porcentagem) para variáveis categóricas.

ATPC = angioplastia transluminal percutânea coronária; EuroSCORE = European System for Cardiac Operative Risk Evaluation; IMC = índice de massa corporal; NYHA = New York Heart Association. 


\section{Resultados clínicos e do procedimento}

A artéria femoral foi a via de acesso em 29 pacientes e a artéria subclávia esquerda, em 2 pacientes. O implante da prótese aórtica CoreValve foi possível em $100 \%$ dos pacientes. Em geral, todos os pacientes apresentaram boa tolerância no momento do implante. O tempo total do procedimento foi de 102,3 $\pm 28,5$ minutos (60-168 minutos) e o tempo de liberação da prótese foi de 8,16 $\pm 3,4$ minutos (mínimo de 4 minutos e máximo de 15).

A posição da prótese estava correta em todos os pacientes, exceto em um caso em que o implante foi baixo demais e causou regurgitação periprotética grave, confirmada por angiografia e ecocardiograma, requerendo o implante de uma segunda prótese aórtica CoreValve acima da original, com excelente resultado. Pós-dilatação da válvula foi realizada em 6 (19,3\%) pacientes. O gradiente máximo caiu de 65,9 $\pm 20,5 \mathrm{mmHg}$ (30-110 $\mathrm{mmHg}$ ) para 0,48 $\pm 1,50 \mathrm{mmHg}(0-5 \mathrm{mmHg})$ $(\mathrm{P}<0,0001)$. Nenhum paciente teve insuficiência aórtica residual $>$ grau 2 de Sellers. O fechamento percutâneo do acesso vascular foi bem-sucedido em 30 (96,8\%) casos. Após o procedimento, 10 (31\%) pacientes necessitaram de marca-passo definitivo, pela presença de bloqueio atrioventricular (Tabela 2).

\section{Complicações durante o procedimento}

Houve complicações do procedimento em 3 (9,6\%) pacientes. Não houve casos de dispositivo obstruindo as artérias coronárias ou acidente vascular cerebral. Complicações vasculares que necessitaram cirurgia de emergência foram observadas em $2(6,4 \%)$ pacientes (em um, por falha do dispositivo de fechamento percutâneo, e no outro, por ruptura ilíaca), tendo um desses pacientes, que era imunodeprimido com agamaglobulinemia comum, morrido 19 dias após o implante, em choque séptico e com infecção da ferida cirúrgica. Um paciente com cirurgia de revascularização coronária prévia, com enxerto permeável da artéria mamária interna esquerda para a artéria descendente anterior, apresentou infarto do miocárdio decorrente de dissecção da artéria mamária durante o procedi-

TABELA 2

Dados de acompanhamento $(n=31)$

\begin{tabular}{lc}
\hline Evento cardiovascular & $2(6,4 \%)$ \\
Mortalidade hospitalar & $1(3,2 \%)$ \\
Acidente vascular cerebral & $\overline{(3,2 \%)}$ \\
Infarto do miocárdio & $2(6,4 \%)$ \\
Complicações vasculares & \\
Ruptura/bloqueio cardíaco & $10 \overline{(31 \%)}$ \\
Implante de marca-passo definitivo & $4(13,3 \%)$ \\
Mortalidade $\leq 1$ mês & $5(16,1 \%)$ \\
Mortalidade geral & \\
\hline
\end{tabular}

mento (realizado pela artéria subclávia esquerda), que foi tratado com sucesso com implante de stent.

\section{Acompanhamento dos resultados clínicos}

O tempo médio de hospitalização após o procedimento foi de 8,1 $\pm 3,9$ dias (4-20 dias). O gradiente transvalvar aórtico máximo medido por ecocardiograma 72 horas após o procedimento caiu de $76,6 \pm 18,0 \mathrm{mmHg}$ para 15,8 $\pm 6,9 \mathrm{mmHg}$ (Figura 4). A mortalidade geral e a taxa de eventos cardiovasculares maiores após 30 dias foram de 3,2\% e 9,6\%, respectivamente. Em relação à classe funcional, observou-se notável alívio dos sintomas antes da alta, com queda média da classe funcional da New York Heart Association de 3,2 $\pm 0,6$ antes para 1,2 $\pm 0,4$ após o procedimento $(\mathrm{P}<0,0001)$. Após acompanhamento médio de $154 \pm 90$ dias, foram registradas 4 mortes, 1 morte súbita e 3 por causas não-cardíacas (Figura 5).

\section{DISCUSSÃO}

Os resultados deste estudo confirmam dados publicados anteriormente demonstrando que o implante

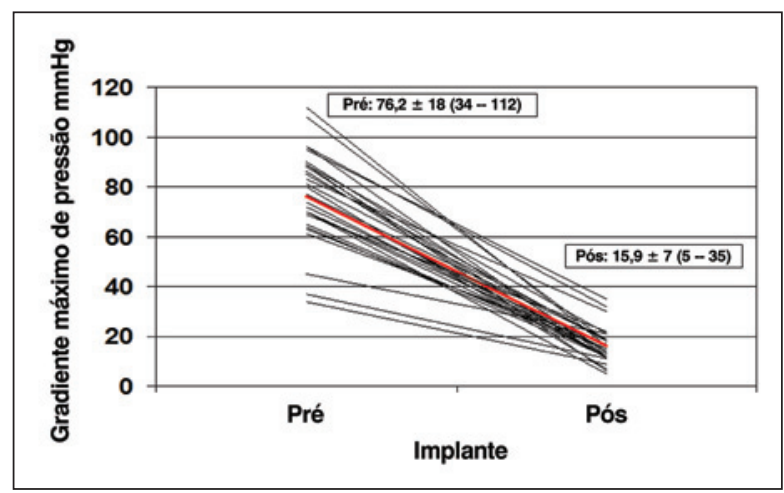

Figura 4 - Medidas ecocardiográficas: gradiente máximo da válvula aórtica $(\mathrm{mmHg})$ antes e após implante da prótese aórtica CoreValve.

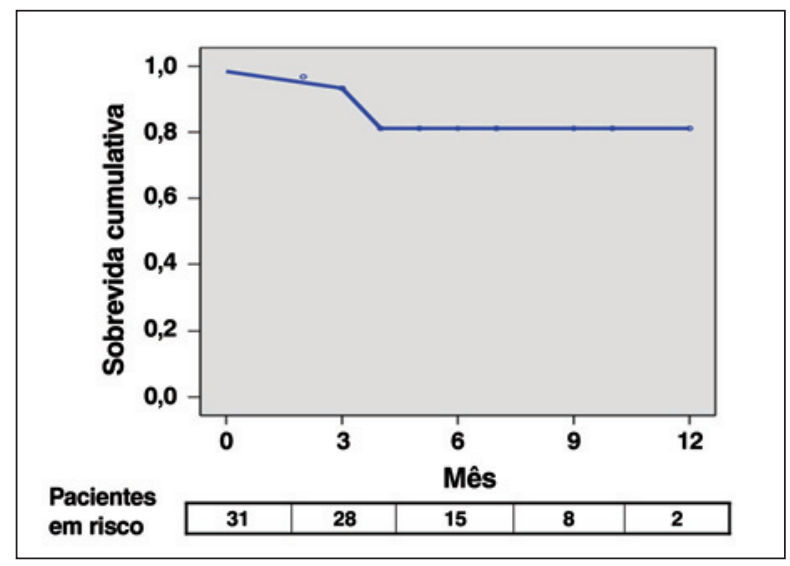

Figura 5 - Estimativa de sobrevida da população do estudo ( $n=31$ ), utilizando a curva de sobrevida de Kaplan-Meier. 

Aórtica Sintomática Grave. Rev Bras Cardiol Invas. 2009;17(2):176-82.

percutâneo de válvula aórtica em pacientes com estenose aórtica grave com alto risco cirúrgico é possível, com elevada taxa de sucesso ${ }^{9-14}$ e baixa taxa de complicações, com resultados semelhantes aos obtidos por Sarmento-Leite et al. ${ }^{15}$. Além disso, em nossa série descrevemos um caso bem-sucedido de implante de válvula aórtica percutânea utilizando a prótese aórtica CoreValve para estenose aórtica em um paciente com prótese biológica disfuncional implantada três anos antes. Esse caso reflete a viabilidade e a segurança do procedimento. Como uma nova cirurgia está associada a elevadas taxas de morbidade e mortalidade (aproximadamente $15 \%$ ), a possibilidade de tratamento percutâneo para disfunção protética, decorrente de insuficiência grave $^{16}$ ou estenose, é uma alternativa terapêutica para esse subgrupo de pacientes.

O acesso femoral foi utilizado na maioria dos pacientes, mas, apesar dos avanços nessa técnica, que inclui a redução do diâmetro dos dispositivos para liberação da prótese (de $24 \mathrm{~F}$ para $18 \mathrm{~F}$ no caso da prótese aórtica CoreValve $)^{9}$, essa abordagem foi contraindicada em alguns pacientes com doença da artéria periférica ilíaco-femoral grave. Para superar essa limitação, a via transapical foi desenvolvida para a prótese Edwards-Sapiens, embora implicando toracotomia esquerda, e os resultados descritos até o momento sugerem maior mortalidade que para a abordagem femoral, embora os pacientes tivessem EuroSCORE mais alto ${ }^{17,18}$.

O acesso vascular em dois de nossos pacientes foi a artéria subclávia esquerda para implantar a prótese aórtica CoreValve, após descartada a abordagem femoral. A subclávia esquerda é uma abordagem atraente, como alternativa para a abordagem transapical, para o tratamento de estenose grave com a prótese aórtica CoreValve em pacientes com alto risco cirúrgico e nos quais é impossível utilizar a abordagem femoral. Essa abordagem requer a ajuda do cirurgião e pode ser realizada com anestesia local e sedação superficial, embora uma possível complicação dessa abordagem seja a dissecção do enxerto da artéria mamária esquerda, conforme aconteceu em nosso caso. Na série de Grube et al. ${ }^{19}$, de 136 pacientes, 3 utilizaram a via subclávia, embora os autores não tenham analisado resultados ou complicações. O sucesso inicial do implante $(100 \%)$ foi melhor que o descrito por Grube et al. ${ }^{10}$ (88\%) e semelhante ao de Piazza et al. ${ }^{20}$ (97\%), embora os pacientes deste último tivessem maior risco cirúrgico que os nossos.

A alta taxa de sucesso do procedimento observada em nossa série foi acompanhada por mortalidade hospitalar de 3,2\%, menor que a obtida com o EuroSCORE $(17,5 \%)$. A comparação da mortalidade com o EuroSCORE deve ser feita com cuidado, já que vários estudos demonstram que esse modelo preditivo pode superestimar a mortalidade nesses pacientes ${ }^{21}$.
O número de complicações periprocedimento foi baixo, sendo duas complicações vasculares e uma com infarto agudo do miocárdio. As complicações vasculares foram associadas a alta taxa de morbidade e mortalidade, e, consequentemente, o manuseio cuidadoso do dispositivo e a seleção adequada de pacientes são extremamente importantes para evitar ruptura arterial.

A taxa de implante de marca-passos após o procedimento em nossa série foi alta (31\%) em comparação com outras séries. Em 646 pacientes com estenose aórtica submetidos a implante percutâneo da prótese aórtica CoreValve, a incidência de implante definitivo de marca-passo foi de 9,3\% ${ }^{19}$. Essas porcentagens são mais elevadas que as das séries cirúrgicas, que são de aproximadamente $6 \%$ a $6,5 \%^{22,23}$. Atualmente, não conhecemos os fatores relacionados ao desenvolvimento de bloqueio atrioventricular após implante de prótese aórtica CoreValve, embora a maioria dos pacientes já tivesse distúrbios de condução e a proximidade com o sistema elétrico de condução do coração possa interferir com o posicionamento da prótese. Um estudo recente demonstrou que a necessidade de marca-passo permanente poderia ser prevista com uma combinação de variáveis basais, como bloqueio de ramo esquerdo com desvio do eixo esquerdo, dimensão do septo interventricular $>17 \mathrm{~mm}$ ou espessura da cúspide coronária $>8 \mathrm{~mm}$, com sensibilidade de $75 \%$ e especificidade de $100 \%{ }^{24}$. Em nossa série, um paciente que desenvolveu bloqueio do ramo esquerdo (duração do QRS de 180 mseg) após implante da válvula morreu subitamente durante o acompanhamento. Um modelo como o apresentado por Jilaihawi et al. ${ }^{24}$ poderia ser utilizado para selecionar pacientes para marca-passo profilático, e, dessa forma, simplificar e acelerar o processo de recuperação após o procedimento. É necessário realizar estudos específicos para avaliar esses aspectos e estabelecer as indicações para o implante de marca-passo definitivo nesses pacientes.

Nossos dados demonstram que o implante da prótese aórtica CoreValve soluciona a estenose valvar. Ocasionalmente é necessário pós-dilatar a válvula para otimizar a colocação e a expansão da estrutura da prótese e assim reduzir uma das possíveis causas de regurgitação periprotética. As consequências dessa pós-dilatação e seu efeito a longo prazo sobre a válvula, assim como sua durabilidade, serão objeto de estudos futuros.

\section{Limitações}

Neste estudo analisamos os resultados do implante da prótese aórtica CoreValve em um número limitado de pacientes, com curto período de acompanhamento e sem grupo controle. Acreditamos ser necessária a realização de outros estudos, com maior período de acompanhamento e maior tamanho da amostra, para avaliar a segurança do dispositivo. 


\section{CONCLUSÕES}

O implante percutâneo da prótese aórtica CoreVale como tratamento alternativo para estenose aórtica grave em pacientes com alto risco cirúrgico é viável e seguro, com taxa de sucesso elevada para o procedimento e porcentagem de complicações abaixo do esperado para um risco cirúrgico estimado utilizando-se o EUrOSCORE.

\section{CONFLITO DE INTERESSES}

Os autores declararam inexistência de conflito de interesses.

\section{REFERÊNCIAS BIBLIOGRÁFICAS}

1. Nkomo VT, Gardin JM, Skelton TN, Gottdiener JS, Scott CG, Enriquez-Sarano M. Burden of valvular heart diseases: a population-based study. Lancet. 2006;368(9540):1005-11.

2. Iung B, Baron G, Butchart EG, Delahaye F, Gohlke-Bärwolf C, Levang OW, et al. A prospective survey of patients with valvular heart disease in Europe: the Euro Heart Survey on Valvular Heart Disease. Eur Heart J. 2003;24(13):1231-43.

3. Bonow RO, Carabello BA, Kanu C, Leon AC Jr, Faxon DP, Freed MD, et al. ACC/AHA 2006 guidelines for the management of patients with valvular heart disease: a report of the American College of Cardiology/American Heart Association Task Force on Practice Guidelines (writing committee to revise the 1998 Guidelines for the management of patients with valvular heart disease): developed in collaboration with the Society of Cardiovascular Anesthesiologists: endorsed by the Society for Cardiovascular Angiography and Interventions and the Society of Thoracic Surgeons. Circulation. 2006;114(5):e84-231.

4. Kolh P, Kerzmann A, Honore C, Comte L, Limet R. Aortic valve surgery in octogenarians: predictive factors for operative and long-term results. Eur J Cardiothorac Surg. 2007;31(4):600-6.

5. Caballero-Borrego J, Gómez-Doblas JJ, Valencia-Serrano FM, Cabrera-Bueno F, Rodríguez-Bailón I, Sánchez-Espín G, et al. Influence of sex on perioperative outcomes in patients undergoing valve replacement for severe aortic stenosis. Rev Esp Cardiol. 2009;62(1):31-8.

6. Melby SJ, Zierer A, Kaiser SP, Guthrie TJ, Keune JD, Schuessler $\mathrm{RB}$, et al. Aortic valve replacement in octogenarians: risk factors for early and late mortality. Ann Thorac Surg. 2007; 83(5):1651-7.

7. Iung B, Cachier A, Baron G, Messika-Zeitoun D, Delahaye F, Tornos P, et al. Decision-making in elderly patients with severe aortic stenosis: why are so many denied surgery? Eur Heart J. 2005;26(24):2714-20.

8. Cribier A, Eltchaninoff $H$, Tron C, Bauer F, Agatiello C, Nercolini D, et al. Treatment of calcific aortic stenosis with the percutaneous heart valve: mid-term follow-up from the initial feasibility studies: the French experience. J Am Coll Cardiol. 2006;47(6):1214-23.

9. Grube E, Laborde JC, Gerckens U, Felderhoff T, Sauren B, Buellesfeld L, et al. Percutaneous implantation of the CoreValve self-expanding valve prosthesis in high-risk patients with aortic valve disease: the Siegburg first-in-man study. Circulation. 2006;114(15):1616-24.

10. Grube E, Schuler G, Buellesfeld L, Gerckens U, Linke A, Wenaweser $\mathrm{P}$, et al. Percutaneous aortic valve replacement for severe aortic stenosis in high-risk patients using the second- and current third-generation self-expanding CoreValve prosthesis: device success and 30-day clinical outcome. J Am Coll Cardiol. 2007;50(1):69-76.

11. Zegdi R, Sleilaty G, Lafont A, Fabiani JN. Percutaneous aortic valve replacement with CoreValve prosthesis. J Am Coll Cardiol. 2008;51(2):170.

12. Webb JG, Pasupati S, Humphries K, Thompson C, Altwegg $L$, Moss R, et al. Percutaneous transarterial aortic valve replacement in selected high-risk patients with aortic stenosis. Circulation. 2007;116(7):755-63.

13. García E, Pinto AG, Sarnago Cebada F, Pello AM, Paz M, García-Fernández MA, et al. Percutaneous aortic valve implantation: initial experience in Spain. Rev Esp Cardiol. 2008; 61(11):1210-4.

14. Moreno R, Calvo L, Filgueiras D, López T, Sánchez-Recalde A, Jiménez-Valero $S$, et al. Percutaneous implantation of aortic valve prosthesis in patients with symptomatic severe aortic stenosis rejected for surgical valve replacement. Rev Esp Cardiol. 2008;61(11):1215-9.

15. Sarmento-Leite R, Quadros AS, Prates PRL, Zanatta LG, Salgado Filho PA, Grando T, et al. Implante valvular aórtico percutâneo: experiência inicial do sul do Brasil. Rev Bras Cardiol Invas. 2008;16(4):398-405.

16. Wenaweser P, Buellesfeld L, Gerckens U, Grube E. Percutaneous aortic valve replacement for severe aortic regurgitation in degenerated bioprosthesis: the first valve in valve procedure using the CoreValve Revalving system. Catheter Cardiovasc Interv. 2007;70(5):760-4.

17. Lichtenstein SV, Cheung A, Ye J, Thompson CR, Carere RG, Pasupati S, et al. Transapical transcatheter aortic valve implantation in humans: initial clinical experience. Circulation. 2006;114(6):591-6.

18. Walther T, Simon P, Dewey T, Wimmer-Greinecker G, Falk $\mathrm{V}$, Kasimir MT, et al. Transapical minimally invasive aortic valve implantation: multicenter experience. Circulation. 2007; 116(11 Suppl):I240-5.

19. Grube E, Buellesfeld L, Mueller R, Sauren B, Zickmann B, Nair D, et al. Progress and current status of percutaneous aortic valve replacement: results of three device generations of the CoreValve Revalving system. Cir Cardiovasc Intervent. 2008;1:167-75.

20. Piazza N, Grube E, Gerckens U, den Heijer P, Linke A, Luha $\mathrm{O}$, et al. Procedural and 30-day outcomes following transcatheter aortic valve implantation using the third generation $(18 \mathrm{Fr})$ CoreValve Revalving system: results from the multicentre, expanded evaluation registry 1 -year following CE mark approval. Eurolntervention. 2008;4(2):242-9.

21. Osswald BR, Gegouskov V, Badowski-Zyla D, Tochtermann $U$, Thomas G, Hagl S, et al. Overestimation of aortic valve replacement risk by EuroSCORE: implications for percutaneous valve replacement. Eur Heart J. 2009;30(1):74-80.

22. Dawkins S, Hobson AR, Kalra PR, Tang AT, Monro JL, Dawkins KD. Permanent pacemaker implantation after isolated aortic valve replacement: incidence, indications, and predictors. Ann Thorac Surg. 2008;85(1):108-12.

23. Koplan BA, Stevenson WG, Epstein LM, Aranki SF, Maisel WH. Development and validation of a simple risk score to predict the need for permanent pacing after cardiac valve surgery. J Am Coll Cardiol. 2003;41(5):795-801.

24. Jilaihawi $H$, Chin $D$, Vasa-Nicotera $M$, Jeilan $M$, Spyt $T$, Ng $\mathrm{GA}$, et al. Predictors for permanent pacemaker requirement after transcatheter aortic valve implantation with the CoreValve bioprosthesis. Am Heart J. 2009;157(5):860-6. 